Anaesthesist 2021 · 70:515-521 https://doi.org/10.1007/s00101-021-00928-5 Angenommen: 27. Januar 2021 Online publiziert: 23. Februar 2021 (c) Der/die Autor(en) 2021
Isabell Pekrul ${ }^{1}$ Thomas Schachtner ${ }^{2} \cdot$ Bernhard Zwißler $^{3} \cdot$ Patrick Möhnle $^{1}$

' Klinik für Anaesthesiologie, Abteilung für Transfusionsmedizin, Zelltherapeutika und Hämostaseologie, Universität München (LMU), München, Deutschland

${ }^{2}$ Anästhesie und Intensivmedizin, Schön Klinik München Harlaching, München, Deutschland

${ }^{3}$ Klinik für Anaesthesiologie, Universität München (LMU), München, Deutschland

\title{
Tranexamsäure zur Blutungsprophylaxe bei Trauma und orthopädischen Eingriffen - Standard oder individualisierte Anwendung?
}

Seit Langem ist der Einsatz von Tranexamsäure bei der Therapie von Blutungen, vorrangig bei Hyperfibrinolyse, sowie zur Prophylaxe von Blutungen in definierten Indikationsbereichen wie kardiochirurgischen Eingriffen etabliert. In den letzten Jahren hat der Gebrauch von Tranexamsäure zur Blutungsprophylaxe als Behandlungsstandard in breiten Patientenkollektiven deutlich zugenommen. Vor allem bei Trauma sowie bei orthopädischen Eingriffen erfolgt zunehmend ein prophylaktischer Einsatz als Teil der klinischen Routine. Mit diesem Einsatzbereich des Präparates und dem damit verbundenen Wandel der Indikationen treten jedoch regelhaft folgende Fragen auf: 1) Ist ein breiter, nichtselektionierter Einsatz im Bereich der Blutungsprophylaxe mit einem Anstieg von Nebenwirkungen verbunden und 2) handelt es sich beim prophylaktischen Einsatz des Präparates um einen „Off Label Use“ bzw. sind beim prophylaktischen Einsatz ggf. besondere rechtliche Vorgaben z.B. im Rahmen der Aufklärung zu beachten?

\section{Historie und Wirkprinzip}

Tranexamsäure (TXA) als Antifibrinolytikum wurde 1962 erstmals beschrieben, ursprünglich mit dem Ziel, ein Präparat zur Behandlung peripartaler Blutungen zur Verfügung stellen zu können [56]. Tranexamsäure ist strukturell der Ami- nosäure Lysin ähnlich. Der Wirkmechanismus von Tranexamsäure beruht auf der Bindung des Präparates an den Lysinrezeptor von Plasminogen, hierdurch wird die Aktivierung von Plasminogen durch Plasminogenaktivatoren („tissue plasminogen activator", t-PA) verhindert, was in der Folge die Auflösung von Gerinnseln durch Plasmin einschränkt [33]. Einer Hyperfibrinolyse wird somit pharmakologisch entgegengewirkt; bei aufrechterhaltener Balance zwischen Gerinnselbildung und Lyse trägt TXA durch Einschränkung der Fibrinolyse zu einer Stabilisierung des Gerinnsels bei. Die Effektstärke bei der Behandlung von Blutungen ist abhängig von der klinischen Situation; bei leichten Blutungen ist ggf. eine alleinige Therapie mit TXA ausreichend, bei schweren Blutungskomplikationen ist die Therapie mit TXA in der Regel als begleitend zur Substitution zellulärer und plasmatischer Produkte zu betrachten.

Eine intravenöse, perorale sowie topische Gabe ist möglich; aufgrund der vorwiegend renalen Ausscheidung ist eine Dosisreduktion bei schwerer Niereninsuffizienz zu beachten; das Nebenwirkungsprofil ist insgesamt günstig; gastrointestinale Beschwerden können (v. a. bei oraler Anwendung) häufiger ( $\geq 1 / 100$ bis $<1 / 10$ ) auftreten; in unbekannter Häufigkeit sind neben allergischen Reaktionen auch Sehstörungen und Kreislaufreaktio- nen beschrieben. Eine potenziell schwerwiegende Nebenwirkung des Präparates, das Auftreten zerebraler Krampfanfälle, ist im Wesentlichen dosisabhängig und v. a. bei Anwendung in hoher Dosierung in der Kardiochirurgie beschrieben [31]. Auf das potenzielle Risiko thrombembolischer Komplikationen wird unten detailliert eingegangen. Die Zulassung der TXA umfasst die Prophylaxe und Behandlung von Blutungen aufgrund einer generalisierten oder lokalen Hyperfibrinolyse bei Erwachsenen und Kindern ab 1 Jahr. Tranexemsäure hat einen über Jahrzehnte etablierten Stellenwert als Medikation bzw. supportive Medikation bei Menorrhagie sowie im Falle von Blutungskomplikationen bei Hämophilie, Von-Willebrand-Syndrom und weiteren seltenen Faktorenmängeln [8]. Die Anwendung bei gastrointestinaler Blutung ist nach einer aktuellen Studie, welche keinen positiven Effekt von TXA bei akuter gastrointestinaler Blutung aufzeigen konnte, umstritten [9]. Seit 2010 wurden über die lange bekannten Einsatzbereiche hinaus weitere Indikationen der TXA beschrieben und teils mit Daten großer multizentrischer klinischer Studien belegt, v. a. der Einsatz bei Trauma (CRASH-2) sowie bei peripartaler Blutung (WOMAN-Trial) [10, 58]; in beiden Indikationsbereichen ist die Substanz mittlerweile in Leitlinien und Empfehlungen aufgenommen. In den letzten Jah- 
ren wurden zudem zunehmend Studien und Empfehlungen zum prophylaktischen perioperativen Einsatz insbesondere im Rahmen von Patient-Blood-Management-Konzepten veröffentlicht.

\section{Tranexamsäure bei Trauma und Schädel-Hirn-Trauma}

Die CRASH-2-Studie ist die bislang größte Untersuchung (multizentrisch, international, 20.211 Patienten) in dieser Indikation; sie zeigte einen Vorteil einer frühen Gabe (innerhalb $3 \mathrm{~h}$ nach Trauma) in Bezug auf das Überleben bei starkem Blutverlust oder Gefahr schwerer Blutungen („number needed to treat“ in Bezug auf Letalität aufgrund Blutung: 119), jedoch auch einen nachteiligen Effekt bei zu später Verabreichung $(>3 \mathrm{~h}$ nach Trauma) [10]. Es wurde keine erhöhte Rate an arteriellen oder venösen thrombembolischen Ereignissen (ATE/VTE) in der mit TXA behandelten Gruppe beobachtet [10]. Einschränkend bei der Bewertung des Risikos thrombembolischer Ereignisse in der CRASH-2-Studie ist jedoch die Tatsache, dass solche Ereignisse zwar per Studienprotokoll registriert wurden, die Patienten aber nicht standardisiert in Bezug auf diese Nebenwirkung untersucht wurden (,recorded only when clear clinical evidence") und somit ein ,underreporting" thrombembolischer Komplikationen nicht auszuschlieBen ist [10]. Interessanterweise zeigte sich kein Unterschied in der Transfusionshäufigkeit zwischen der mit TXA und der ohne TXA behandelten Patientengruppe [45]. Trotz Kritik an methodischen Schwächen der Studie $[4,38]$ sowie Hinweisen auf eine erhöhte Inzidenz von VTE in anderen Studien $[22,37,57]$ etablierte sich die Dosierung von $1 \mathrm{~g}$ TXA als i.v.-Bolus und einer kontinuierlichen Gabe von $1 \mathrm{~g}$ i.v. über $8 \mathrm{~h}$ in verschiedenen Leitlinien und Empfehlungen $[6,39$, $43,51]$ im Sinne einer frühen bzw. im engeren Sinne prophylaktischen Gabe bei schwerem Trauma.

Tranexamsäure soll in dieser Indikation einer überschießenden Hyperfibrinolyse entgegenwirken: Dieses Therapieprinzip bei Trauma ist jedoch nicht unumstritten. Kritiker argumentieren, dass die Ausprägung einer systemischen
Hyperfibrinolyse von einem "fibrinolytic shutdown", der durch das Gewebstrauma induziert wird, klinisch auch anhand von Scores und Verletzungsschwere nicht zu unterscheiden ist und eine präemptive TXA-Gabe bei Patienten mit "fibrinolytic shutdown" zu einer Erhöhung des Risikos für thrombembolische Komplikationen und Multiorganversagen führen könnte [35]. Um dieses Risiko zu minimieren, wurde in kleineren Studien der Versuch unternommen, TXA nach viskoelastischen Messverfahren (VEM) gesteuert zu verabreichen, jedoch fand sich nur eine schwache Korrelation zwischen VEM und der nach „Goldstandard“ mittels PAP-Komplexen gemessener Hyperfibrinolyse [15, 42]. Interessanterweise zeigten jedoch eine weitere prospektive Studie sowie eine Propensity-Score-gematchte Studie, die beide bei Aufnahme im Schockraum die Auswirkung einer frühen TXA-Gabe am Unfallort mittels VEM untersuchten, eine höhere Gerinnselstabilität und einen geringeren D-Dimer-Anstieg mit TXA; dies unterstreicht den potenziellen Stellenwert einer prophylaktischen Gabe bei Traumapatienten $[28,52]$.

Die CRASH-3-Studie (,international, multicenter, randomised cotrolled trial ${ }^{\text {" }}$ (RCT), $n=12.797$ ) fokussierte auf Patienten mit isoliertem Schädel-Hirn-Trauma (SHT). Sie erbrachte den Nachweis einer geringeren Früh- und Gesamtletalität bei Patienten mit leichtem bis mittelgradigem SHT und TXA-Gabe beim Einsatz entsprechend dem Dosisregime der CRASH-2-Studie. Dabei gab es im Vergleich zur Placebogruppe keinen Unterschied im neurologischen Outcome (Glasgow Outcome Scale), in der Häufigkeit von Krampfanfällen sowie in der Anzahl von VTE-Komplikationen [11]. Ebenso konnte eine aktuelle Metaanalyse Vorteile für den frühzeitigen Einsatz $(<3 \mathrm{~h})$ von TXA hinsichtlich einer geringeren Letalität und einer geringeren intrazerebralen Hämatomausbreitung bei gleicher Häufigkeit an neurochirurgischen Interventionen zeigen [23].

Zusammenfassend besteht in Leitlinien Konsens für eine frühzeitige Gabe von TXA bei Traumapatienten; Ergebnisse von weiteren Studien zur idealen Dosierung und zum optimalen Zeitpunkt der Gabe sind noch ausstehend. Eine vorab durchgeführte VEM-Testung wird nicht empfohlen [51]. Angesichts einer möglicherweise erhöhten VTE-Inzidenz unter TXA ist jedoch der Stellenwert einer VTE-Prophylaxe mit physikalischen und ggf. medikamentösen Maßnahmen im späteren Verlauf zu betonen [2].

\section{Prophylaktische Anwendung in der Orthopädie}

In den letzten Jahren wird TXA v. a. bei elektiven endoprothetischen Knie- und Hüftgelenkoperationen (KTEP/HTEP) auch zunehmend als Prophylaxe zur Reduktion von Blutungen eingesetzt. Allerdings wird die TXA-Applikation in diesem Anwendungsbereich sowohl innerhalb der Fachdisziplinen als auch zwischen anästhesiologischer und orthopädischer Fachdisziplin durchaus kontrovers diskutiert. Befürworter einer prophylaktischen TXA-Gabe führen als Argumente reduzierte Blutungsvolumina sowie eine reduzierte Transfusionswahrscheinlichkeit, aber auch eine verkürzte Erholungszeit und reduzierte Inflammation ins Feld; Kritiker argumentieren mit einem potenziell erhöhten Risiko für thrombembolische Komplikationen. Durch die zunehmende Fülle und Heterogenität an Studien und Metaanalysen der letzten Jahre im Hinblick auf unterschiedliche Einschlusskriterien, Dosisregime, Applikationsrouten und Endpunkte erscheint eine Konsensfindung erschwert.

\section{Effektstärke}

Als primäre Outcome-Parameter werden in den kontrollierten Studien meist die Reduktion der Transfusionswahrscheinlichkeit und des perioperativen Blutverlustes genannt. Die Vermeidung der allogenen Bluttransfusion stellt ein Kernelement vieler „Enhanced-recovery-after-surgery"(ERAS)-Programme dar; für die Fremdblutgabe wurden Assoziationen mit einem erhöhten Infektionsrisiko und einer Verlängerung der Krankenhausverweildauer als auch mit einer erhöhten Mortalität gezeigt [3, 26]. Die Inzidenz der allogenen Fremdbluttransfusion ist allerdings sehr 
variabel: Es werden z. B. Inzidenzen von 2,5-35,3\% für KTEP- und 14-29,8\% für HTEP-Operationen berichtet [50]. Neben operationstechnischen Unterschieden sind weitere unabhängige Risikofaktoren für eine Fremdblutgabe gut beschrieben und umfassen beispielsweise (1) die unbehandelte, präoperative Anämie, welche mit einer Prävalenz von ca. $14-23 \%$ beobachtet wird $[29,34]$ sowie (2) verlängerte Operationszeiten [7, 46]. Darüber hinaus nehmen auch unterschiedliche Transfusionstrigger sowie das Flüssigkeits- und Volumenmanagement einen Einfluss. Die Messung bzw. Schätzung des Blutverlustes stellt ebenso eine Herausforderung dar und unterliegt verschiedenen Limitationen, die meist zu einer Überschätzung des Blutverlustes führen [20]. Die Variabilität der Effektstärke zwischen einzelnen Studien ist hierdurch erklärbar. Trotz dieser hohen Variabilität konnte der blutsparende Effekt der prophylaktischen TXA-Gabe jedoch bei verschiedenen chirurgischen Eingriffen, in verschiedenen Gesundheitssystemen und Ethnien reproduziert werden. Einen Anhalt zur Abschätzung der Effektstärke bei operativen Eingriffen in unterschiedlichen Disziplinen bietet die Metaanalyse von Ker et al., die eine Reduktion des Blutverlustes und der Transfusion bis zu einem Drittel nach TXA-Gabe aufzeigen konnte (• Tab. 1; [25]).

Die Unterstützung der Antifibrinolyse mittels TXA vermag durch die Verbesserung der Gerinnselstabilität die prokoagulatorische Seite des Blutgerinnungssystems für den Zeitraum der Operation zu unterstützen; speziell bei HTEP-Operationen gibt es Hinweise auf eine mögliche Induktion einer lokalen Fibrinolyse durch das operative Trauma $[5,13]$. Für den Anwendungsbereich „Endoprothesenchirurgie" wird die blutsparende Wirkung der TXA-Applikation in Metaanalysen in relevanter Größenordnung beschrieben $[1,17,18,36,53,62]$ z. B. eine signifikante Reduktion der Transfusionsrate auf $8,2 \%$ (vs. 19,5\% bei Placebo) und des Blutverlustes von 1089,6 $\pm 1251,5 \mathrm{ml}$ (vs. 1410,3 $\pm 1111,0 \mathrm{ml}$ bei Placebo) [36]. Auf Basis dieser Daten sprechen sich zwei Fachgesellschaften für die Anwendung

Anaesthesist 2021 · 70:515-521 https://doi.org/10.1007/s00101-021-00928-5

(c) Der/die Autor(en) 2021

I. Pekrul · T. Schachtner · B. Zwißler · P. Möhnle

Tranexamsäure zur Blutungsprophylaxe bei Trauma und orthopädischen Eingriffen - Standard oder individualisierte Anwendung?

Zusammenfassung

Tranexamsäure hat einen etablierten Stellenwert in der Behandlung von Blutungen, v.a. bei Hyperfibrinolyse. Zunehmend wird TXA auch prophylaktisch bei Trauma und orthopädischen Eingriffen eingesetzt, wobei sich Fragen nach Risiken sowie einem möglichen Off-Label-Einsatz ergeben. Auf Basis der verfügbaren Literatur lässt sich schließen, dass ein prophylaktischer Einsatz des Präparates in diesen Indikationsbereichen vertretbar ist. Jedoch sollte bei Patienten mit erhöhtem Risiko für thrombembolische Ereignisse weiterhin eine individuelle Abwägung von Nutzen und Risiken durchgeführt und dokumentiert werden. Obwohl die Indikationsgebiete des prophylaktischen Einsatzes bei Trauma und orthopädischen Eingriffen nicht spezifisch in der Fachinformation aufgeführt sind, ist die Anwendung unseres Erachtens nicht als Off Label Use zu bewerten.

Schlüsselwörter

Antifibrinolytika · Perioperativ · Off-label-use · Aufklärung · Thromboserisiko

\section{Tranexamic acid for bleeding prophylaxis in orthopedic surgery and trauma-standard or customized therapy?}

\begin{abstract}
The use of tranexamic acid (TXA) is established in the treatment of bleeding, especially of bleeding due to hyperfibrinolysis. In recent years the prophylactic use of TXA in trauma and orthopedic surgery has increased leading to open questions regarding potentially associated risks and a possible classification as off label use. The available literature provides a sound basis for the recommendation that TXA can be used in these indications provided that an individual risk assessment
\end{abstract}

von TXA bei KTEP- und HTEP-Operationen aus $[16,47]$.

\section{Dosierung und Applikationsweg}

Trotz des Konsenses über den Vorteil einer perioperativen TXA-Gabe in Leitlinien und Empfehlungen bestehen Differenzen zu Dosierung und Applikation. Die deutsche Handlungsempfehlung der Arbeitsgemeinschaft Endoprothetik (AE) gibt eine konkrete Dosierempfehlung: (1) die einmalige präoperative i.v.-Gabe von $15 \mathrm{mg} / \mathrm{kgKG}$ oder (2) $2 \mathrm{~g}$ oral $2 \mathrm{~h}$ vor Abruf in den OP und/oder die topische Applikation von weiteren 1-2 g Tranexamsäure. Die amerikanische Fachgesellschaft der Knie- und Hüftchirurgen (AAHKS) hingegen gibt z. B. keine is done in patients with increased risks for thromboembolic complications. Although the prophylactic use of TXA in orthopedic surgery and trauma is not explicitly listed in the product characteristics, it should not be regarded as an off label use.

Keywords Antifibrinolytics · Perioperative · Off-labeluse $\cdot$ Aufklärung $\cdot$ Thromboembolic risk spezifische Dosisempfehlung, empfiehlt aber eine Einmaldosis unabhängig vom Applikationsweg; sofern eine i.v.-Applikation vorgesehen ist, sollte diese präoperativ erfolgen [16, 47].

\section{Sicherheit}

KTEP- und HTEP-Operationen sind mit einem Risiko für thrombembolische Komplikationen verbunden. Es besteht die Sorge, dass die antifibrinolytische Wirkung von TXA neben anderen bekannten transienten Risikofaktoren wie (1) Operationsdauer, (2) operationstechnischen Aspekten (z. B. Anwendung von Tourniquets), (3) Akute-Phase-Reaktion oder (4) längere Immobilisierungsphasen einen zusätzlichen risikoerhöhenden 
Tab. 1 Abschätzung der Effektstärke bei perioperativer Gabe von TXAin unterschiedlichen operativen Disziplinen, Metaanalyse zum Effekt der Tranexamsäure auf das Risiko von Bluttransfusionen. (Aus Ker et al. [25])

\begin{tabular}{|c|c|c|c|c|c|}
\hline \multirow{2}{*}{$\begin{array}{l}\begin{array}{l}\text { Art der Opera- } \\
\text { tion }\end{array} \\
\text { Herzchirurgie }\end{array}$} & \multirow{2}{*}{$\begin{array}{l}\text { Anzahl der Ereignis- } \\
\text { se (Tranexamsäure/ } \\
\text { Kontrolle) } \\
622 / 835\end{array}$} & \multirow{2}{*}{$\begin{array}{l}\text { Gepooltes Risiko- } \\
\text { Verhältnis (95 \% KI) }\end{array}$} & \multirow{2}{*}{$\begin{array}{l}\text { p-Wert } \\
\\
\\
<0,001\end{array}$} & \multicolumn{2}{|c|}{$\begin{array}{l}\text { Heteroge- } \\
\text { nität }\end{array}$} \\
\hline & & & & 60 & $<0,001$ \\
\hline $\begin{array}{l}\text { Orthopädische } \\
\text { Operationen }\end{array}$ & $298 / 462$ & $0,55(0,49-0,61)$ & $<0,001$ & 83 & $<0,001$ \\
\hline Leberchirurgie & $29 / 54$ & $0,52(0,39-0,68)$ & $<0,001$ & 93 & $<0,001$ \\
\hline $\begin{array}{l}\text { Urologische } \\
\text { Operationen }\end{array}$ & $40 / 60$ & $0,66(0,48-0,91)$ & 0,01 & 2 & 0,31 \\
\hline Gefäßchirurgie & $11 / 19$ & $0,58(0,34-0,99)$ & 0,05 & - & - \\
\hline $\begin{array}{l}\text { Gynäkologische } \\
\text { Operationen }\end{array}$ & $17 / 50$ & $0,86(0,48-1,54)$ & 0,61 & 65 & 0,06 \\
\hline $\begin{array}{l}\text { Schädel- und } \\
\text { Kieferchirurgie }\end{array}$ & $52 / 76$ & $0,63(0,45-0,86)$ & 0,004 & 46 & 0,12 \\
\hline${ }^{\mathrm{a} T e s t}$ auf Effekt & & & & & \\
\hline
\end{tabular}

Effekt auf die Auftretenswahrscheinlichkeit von VTE ausüben könnte [24, 61]. Während nach früheren Daten die Inzidenz der tiefen Beinvenenthrombose (TVT) bei Endoprothesenoperationen mit bis zu $60 \%$ beobachtet wurde, sinkt über die letzten Jahrzehnte das prozedurbezogene TVT-Basisrisiko [2]. Dieser Effekt ist einerseits auf den standardisierten Einsatz der medikamentösen und physikalischen Thromboseprophylaxe zurückzuführen, andererseits führt die Ausrichtung aller orthopädischen und anästhesiologischen Prozesse auf die Umsetzbarkeit einer stringenten Frühmobilisierung innerhalb von $24 \mathrm{~h}$ nach der Operation zur weiteren Senkung der TVT-Rate (z. B. 0,4\% in einer prospektiven Observationsstudie an 17.582 Eingriffen) [14, 21, 40, 48, 49]. Hierzu kann jedoch auch die prophylaktische TXA durch eine Verminderung der lokalen Schwellung und Erleichterung der Frühmobilisierung und Rehabilitation beitragen [12, 27, 44]. Ergebnisse von Metaanalysen zeigen überwiegend keine signifikante Erhöhung des TVTRisikos nach TXA-Gabe bei endoprothetischen Eingriffen [18, 53-55, 60]. Diese Aussage ist jedoch mit Limitationen behaftet, aufgrund der Heterogenität der Studienprotokolle, der statistischen Unterpowerung der RCT hinsichtlich dieses Ereignisses und eines möglichen Publikationsbias. Schließlich sind auch durchaus gegenteilige Ergebnisse publiziert, die eine erhöhte Rate an VTE in der TXA-Gruppe (vs. Placebo; 4,04 vs. $1,71 \%$ ) beschreiben [36]: In diese Metaanalyse von Moskal et al. wurde jedoch eine Studie eingeschlossen, welche sowohl im Placeboarm als auch in den 4 Therapiearmen eine ungewöhnlich hohe TVT-Rate von 9 bis 13\% aufwies [19]. Auf Basis aller vorliegenden Daten fällt die Risiko-Nutzen-Bewertung jedoch insgesamt günstig für die Anwendung von TXA aus, sofern die Patienten kein erhöhtes Risiko für ATE und/oder VTE aufweisen.

Die prophylaktische TXA-Gabe wird neben der Endoprothetik zunehmend auch bei anderen großen orthopädischen Eingriffen mit relevantem Blutungsrisiko eingesetzt. Bei Wirbelsäuleneingriffen wurde eine Reduktion des perioperativen Blutverlustes und Transfusionsrisikos (relatives Risiko 0,65; 95\%-Konfidenzintervall; 0,53-0,85) gezeigt [32, 59]. In der Mehrzahl der Studien wird angesichts längerer Operationszeiten im Vergleich zur Endoprothesenchirurgie nach der präoperativen Bolusgabe von 10-30 mg/ kgKG eine Erhaltungsdosis von 1-2 mg/ kgKG und h gegeben [32]. Bewertet man andererseits das VTE-Risiko bei diesen Eingriffen, sollten die oftmals längeren Operationszeiten, spezielle Lagerungstechniken sowie Einschränkungen der postoperativen Mobilisierbarkeit als potenzielle Risikofaktoren berücksichtigt werden.

\section{Einsatz bei erhöhtem Risiko \\ für thrombembolische Komplikationen}

Gemäß Fachinformation sind thrombembolische Erkrankungen und/oder Risikofaktoren für thrombembolische Ereignisse Kontraindikationen für die Anwendung von TXA. Bei thrombophilen Hochrisikopatienten darf gemäß Fachinformation „Tranexamsäure nur bei strenger Indikationsstellung nach Rücksprache mit einem hämostaseologisch erfahrenen Arzt und unter engmaschiger medizinischer Überwachung angewendet werden." Dies ist v.a. bei einer evtl. Anwendung innerhalb von perioperativen Standards $\mathrm{zu}$ bedenken, da dies auch eine Anamnese für thrombembolische Ereignisse voraussetzt, welche entsprechend dokumentiert vorliegen sollte.

Eine aktuelle Metaanalyse fokussiert auf Risikopatienten und untersucht hier$\mathrm{zu}$ die Inzidenz von VTE in risikoselektionierten vs. nichtrisikoselektionierten Patientenpopulationen: Die Ergebnisse zeigen zwar keine erhöhte Inzidenz für VTE in den Studiengruppen (TXA systemisch vs. Placebo, RR 0,95 $(0,78-1,15))$, die Autoren verweisen aber auf eine aktuell nichtausreichende Datenlage, um eine Anwendungsempfehlung in diesem Kollektiv geben zu können [60]. Die amerikanischen Fachgesellschaften raten in der praktischen Umsetzung zu einer individualisierten Risiko-NutzenBewertung [16]. Die deutsche Arbeitsgemeinschaft für Endoprothetik empfiehlt, bei Hochrisikopatienten die Gabe von TXA zu vermeiden [47].

Bei Patienten mit Risikofaktoren für thrombembolische Ereignisse ist aus Sicht der Autoren eine Gabe von TXA nicht ausgeschlossen, es ist jedoch eine individuelle Abwägung von Risiko und dem potenziellen Nutzen einer prophylaktischen Gabe dieses Antifibrinolytikums notwendig [30], welche zudem sorgfältig dokumentiert werden sollte. Die Balance von Hämostase und Thromboseneigung unterliegt einer Vielzahl an Einflussfaktoren, welche sich zwischen einzelnen Kliniken deutlich unterscheiden: z.B. durch Unterschiede in Operationstechnik und -dauer, Lagerung, Beginn der Mobilisation, 
Standard zur medikamentösen Thromboseprophylaxe. Aus diesem Grund sind allgemeingültige Empfehlungen für den Einsatz von TXA bei diesen Risikopatienten nicht sinnvoll; hier muss auf der Basis von institutionellen Bedingungen wie auch patientenbezogenen Risikofaktoren individuell entschieden werden.

\section{Prophylaktische Gabe von Tranexamsäure - ein „Off Label Use?"}

Tranexamsäure ist indiziert zu „Prophylaxe und Behandlung von Blutungen aufgrund einer lokalen oder generalisierten Hyperfibrinolyse“ (Zitat Fachinformation „Cyklokapron ${ }^{\circledR}$-Injektionslösung, Fa. Pfizer“ [41]), zudem werden in der Fachinformation Hals-, Nasen-, Ohrenoperationen, gynäkologische Operationen, geburtshilfliche Blutungen und Operationen in Thorax und Bauchraum sowie andere größere chirurgische Eingriffe wie z. B. kardiovaskuläre Operationen aufgeführt. Der Einsatz bei Traumapatienten zur Prophylaxe einer durch Hyperfibrinolyse bedingten Blutung ist aus Sicht der Autoren über diese Zulassung abgedeckt. Der Einsatz zur Prophylaxe von Blutungen bei größeren operativen Eingriffen erscheint gerechtfertigt, auch wenn einzelne operative Verfahren wie z. B. Endoprothetik nicht explizit als Indikationsbereich in der Fachinformation aufgeführt sind.

Zum Begriff der „lokalen Hyperfibrinolyse" in der Fachinformation ist kritisch anzumerken, dass der Nachweis einer Hyperfibrinolyse, insbesondere wenn sie auf den Ort der Verletzung oder das Operationsgebiet beschränkt ist, in der klinischen Routine weder vorgesehen noch einfach durchzuführen ist, dass jedoch eine auch lokalisierte Hyperfibrinolyse bei oben genannten Operationen durchaus auftreten kann [5, 13]; jedoch ist aus Sicht der Autoren bei operativen Eingriffen der Nachweis einer Hyperfibrinolyse generell keine zwingende Vorbedingung für den Einsatz des Präparates innerhalb der Zulassung, da dies über den Passus „Prophylaxe“ abgedeckt ist.
Herr Dr. jur. Elmar Biermann (Justiziar der Deutschen Gesellschaft für Anästhesiologie und Intensivmedizin) bewertet die Fragestellung wie folgt (persönliche Kommunikation an Prof. Zwißler vom 30.07.2019):

Gemäß Fachinformation ist Tranexamsäure indiziert zur Prophylaxe und zur Behandlung von Blutungen aufgrund einer lokalen oder generalisierten Hyperfibrinolyse. Im Folgenden gibt die Fachinformation dann die "genauen" Anwendungsgebiete an. An erster Stelle werden wieder Blutungen aufgrund einer lokalen und generalisierten Hyperfibrinolyse genannt, dann werden beispielhaft drei "Blutungssituationen" umschrieben. In den nächsten Spiegelstrichen werden bestimmte Eingriffe genannt, der vorletzte Spiegelstrich betrifft Operationen an Thorax und Bauchraum sowie andere größere chirurgische Eingriffe wie z.B. kardiovaskuläre Operationen. Der Zusatz „wie z. B. "öfnet den Anwendungsbereich der Tranexamsäure über die beschriebenen Eingriffe hinaus. Hätte die Fachinformation andere größere Operationen ausschließen wollen, dann hätte sie die Beispiele als „ausschließlich“ einleiten oder sonstige Eingriffe als kontraindiziert bezeichnen müssen. Die häufige Verwendung des Hinweises „z.B. “ macht deutlich, dass es zwar um Blutungen aufgrund lokaler oder generalisierter Hyperfibrinolyse geht, dass die Operationen aber gerade nicht abschließend dargestellt sind. Im Ergebnis sehe ich deshalb nicht, dass die Behandlung von Blutungen aufgrund lokaler oder generalisierter Hyperfibrinolyse bei orthopädischen Eingriffen eine Behandlung außerhalb des vom Hersteller definierten Anwendungsgebietes wäre. Insofern stellt sich meines Erachtens die Aufklärungsproblematik unter dem speziellen Aspekt „Off Label Use“ nicht.

Eine dezidierte Aufklärung über einen OffLabel Use wird somit aus Sicht der Autoren bei prophylaktischer Gabe in den oben aufgeführten Indikationen in der Regel nicht benötigt, die Indikation zur Gabe sollte jedoch dokumentiert sein. Eine wesentliche Bedingung für dieses Vorgehen ist jedoch, dass das Präparat nicht bei bekannten Kontraindikationen bzw. nur nach sorgfältiger, individuel- ler Risiko-Nutzen-Abwägung eingesetzt wird.

\section{Fazit für die Praxis}

\section{- Tranexamsäure kann in der Prophy- laxe von Blutungen bei Trauma und orthopädischen Eingriffen eingesetzt werden. \\ - Bei Patienten mit thrombembo- lischen Risikofaktoren sollte eine individuelle Nutzen-Risiko-Abwä- gung unter Berücksichtigung klinik- spezifischer Rahmenbedingungen, wie z. B. Operationsstandards und Standards bei der Mobilisation und Thromboseprophylaxe, erfolgen. \\ - Eine Anwendung von Tranexamsäure in der Prophylaxe von Blutungen bei Trauma und orthopädischen Eingriffen ist nicht als „Off Label Use“ zu werten.}

\section{Korrespondenzadresse}

\section{PD Dr. med. Patrick Möhnle}

Klinik für Anaesthesiologie, Abteilung für Transfusionsmedizin, Zelltherapeutika und Hämostaseologie, Universität München (LMU) Marchioninistr. 15, 81377 München,

Deutschland

patrick.moehnle@med.uni-muenchen.de

Funding. Open Access funding enabled and organized by Projekt DEAL.

\section{Einhaltung ethischer Richtlinien}

Interessenkonflikt. I. Pekrul erhielt Honorare, Forschungsförderung, Reisekosten und Kongressgebühren von den Firmen Bayer AG, Bristol Meyer Squibb, CSL Behring, LFB, Novo Nordisk, Pfizer, Roche, Shire/ Takeda und Sobi. P. Möhnle erhielt Honorare, Reisekosten und Kongressgebühren von den Firmen CSL Behring, Pfizer, Biotest, Novo Nordisk, Roche und Shire/Takeda. T. Schachtner und B. Zwißler geben an, dass kein Interessenkonflikt besteht.

Für diesen Beitrag wurden von den Autoren keine Studien an Menschen oder Tieren durchgeführt. Für die aufgeführten Studien gelten die jeweils dort angegebenen ethischen Richtlinien.

Open Access. Dieser Artikel wird unter der Creative Commons Namensnennung 4.0 International Lizenz veröffentlicht, welche die Nutzung, Vervielfältigung, Bearbeitung, Verbreitung und Wiedergabe in jeglichem Medium und Format erlaubt, sofern Sie den/die ursprünglichen Autor(en) und die Quelle ordnungsgemäß nennen, einen Link zur Creative Commons Lizenz beifügen und angeben, ob Änderungen vorgenommen wurden. 
Die in diesem Artikel enthaltenen Bilder und sonstiges Drittmaterial unterliegen ebenfalls der genannten Creative Commons Lizenz, sofern sich aus der Abbildungslegende nichts anderes ergibt. Sofern das betreffende Material nicht unter der genannten Creative Commons Lizenz steht und die betreffende Handlung nicht nach gesetzlichen Vorschriften erlaubt ist, ist für die oben aufgeführten Weiterverwendungen des $\mathrm{Ma}$ terials die Einwilligung des jeweiligen Rechteinhabers einzuholen.

Weitere Details zur Lizenz entnehmen Sie bitte der Lizenzinformation aufhttp://creativecommons.org/ licenses/by/4.0/deed.de.

\section{Literatur}

1. Alshryda S, Sukeik M, Sarda P et al (2014) A systematic review and meta-analysis of the topical administration of tranexamic acid in total hip and knee replacement. Bone Joint J 96-B:1005-1015

2. Arbeitsgemeinschaft Der Wissenschaftlichen Medizinischen Fachgesellschaften - Ständige Kommission Leitlinien (2015) S3-Leitlinie Prophylaxe der venösen Thromboembolie (VTE)

3. Bernard AC, Davenport DL, Chang PK et al (2009) Intraoperative transfusion of $1 \mathrm{U}$ to $2 \mathrm{U}$ packed red blood cells is associated with increased 30-day mortality, surgical-site infection, pneumonia, and sepsis in general surgery patients. J Am Coll Surg 208:931-937, 937.e931-932 (discussion 938-939)

4. BinzS, Mccollester J, Thomas Setal (2015) CRASH-2 study of tranexamic acid to treat bleeding in trauma patients: a controversy fueled by science and social media.J Blood Transfus 2015:874920

5. Blanie A, Bellamy L, Rhayem Y et al (2013) Duration of postoperative fibrinolysis after total hip or knee replacement: a laboratory follow-up study. Thromb Res 131:e6-e11

6. Bocci MG, Nardi G, Veronesi G et al (2019) Early coagulation support protocol: a valid approach in real-life management of major trauma patients. Results from two Italian centres. Injury 50:1671-1677

7. Bohl DD, Ondeck NT, Darrith B et al (2018) Impact of operative time on adverse events following primary total joint arthroplasty. J Arthroplasty 33:2256-2262.e4

8. Cai J, Ribkoff J, Olson S et al (2020) The many roles of tranexamic acid: an overview of the clinical indications for TXA in medical and surgical patients. Eur J Haematol 104:79-87

9. Collaborators H-IT (2020) Effects of a highdose 24-h infusion of tranexamic acid on death and thromboembolic events in patients with acute gastrointestinal bleeding (HALT-IT): an international randomised, double-blind, placebocontrolled trial. Lancet 395:1927-1936

10. Crash-2 Trial Collaborators (2010) Effects of tranexamic acid on death, vascular occlusive events, and blood transfusion in trauma patients with significant haemorrhage (CRASH-2): a randomised, placebo-controlled trial. Lancet 376:23-32

11. Crash-3 Trial Collaborators (2019) Effects of tranexamic acid on death, disability, vascular occlusive events and other morbidities in patients with acute traumatic brain injury (CRASH-3): a randomised, placebo-controlled trial. Lancet 394:1713-1723

12. Dorweiler MA, Boin MA, Froehle AW et al (2019) Improved early postoperative range of motion in total knee arthroplasty using tranexamic acid: a retrospective analysis. JKnee Surg 32:160-164
13. Eriksson BI, Hultman E, Martinell S et al (1991) Regional fibrinolysis following total hip replacement. Thromb Res 62:441-447

14. Falck-Ytter $Y$, Francis CW, Johanson NA et al (2012) Prevention of VTE in orthopedic surgery patients: antithrombotic therapy and prevention of thrombosis, 9th ed: American college of chest physicians evidence-based clinical practice guidelines. Chest 141:e278S-e325S

15. Faraoni D, Levy JH (2019) Tranexamic acid for acute hemorrhage: when is enough evidence enough? Anesth Analg 129:1459-1461

16. Fillingham $Y A$, Ramkumar DB, Jevsevar DS et al (2018) Tranexamic acid use in total joint arthroplasty: the clinical practice guidelines endorsed by the American Association of Hip and Knee Surgeons, American Society of Regiona Anesthesia and Pain Medicine, American Academy of Orthopaedic Surgeons, Hip Society, and Knee Society. J Arthroplasty 33:3065-3069

17. Fillingham YA, Ramkumar DB, Jevsevar DS et al (2018) The efficacy of tranexamic acid in total hip arthroplasty: a network meta-analysis. J Arthroplasty 33(084):3083-3089.e3

18. Gandhi R, Evans HMK, Mahomed SR et al (2013) Tranexamic acid and the reduction of blood loss in total knee and hip arthroplasty: a meta-analysis. BMC Res Notes 6:184

19. Imai N, Dohmae Y, Suda K et al (2012) Tranexamic acid for reduction of blood loss during total hip arthroplasty. J Arthroplasty 27:1838-1843

20. Jaramillo S, Montane-Muntane M, Capitan D et al (2019) Agreement of surgical blood loss estimation methods. Transfusion 59:508-515

21. Jenny J-Y, Pabinger I, Samama CM (2018) European guidelines on perioperative venous thromboembolism prophylaxis: Aspirin. Eur J Anaesthesiol 35:123-129

22. Johnston LR, Rodriguez CJ, Elster EA et al (2018) Evaluation of military use of tranexamic acid and associated thromboembolic events. JAMA Surg 153:169-175

23. July J, Pranata R (2020) Tranexamic acid is associated with reduced mortality, hemorrhagic expansion, and vascular occlusive events in traumatic brain injury - meta-analysis of randomized controlled trials. BMC Neurol 20:119

24. Kearon C, Kahn SR (2020) Long-term treatment of venous thromboembolism. Blood 135:317-325

25. Ker K, Edwards P, Perel P et al (2012) Effect of tranexamic acid on surgical bleeding: systematic review and cumulative meta-analysis. BMJ 344:e3054

26. Kim JL, Park J-H, Han S-B et al (2017) Allogeneic blood transfusion is a significant risk factor for surgical-site infection following total hip and knee arthroplasty: a meta-analysis. J Arthroplasty 32:320-325

27. Krauss ES, Cronin M, Suratwala SJ et al (2017) Use of intravenous tranexamic acid improves early ambulation after total knee arthroplasty and anterior and posterior total hip arthroplasty. Am J Orthop 46:E314-E319

28. Kunze-Szikszay $N$, Krack LA, Wildenauer $P$ et al (2016) The pre-hospital administration of tranexamic acid to patients with multiple injuries and its effects on rotational thrombelastometry: a prospective observational study in pre-hospita emergency medicine. Scand J Trauma Resusc Emerg Med 24:122

29. Lasocki S, Krauspe R, Heymann CV et al (2015) PREPARE: the prevalence of perioperative anaemia and need for patient blood management in elective orthopaedic surgery: a multicentre, observational study. Eur J Anaesthesiol 32:160-167

30. Lier H, Maegele M, Shander A (2019) Tranexamic acid for acute hemorrhage: a narrative review of landmark studies and a critical reappraisal of its use over the last decade. Anesth Analg 129:1574-1584

31. Lin Z, Xiaoyi Z (2016) Tranexamic acid-associated seizures: a meta-analysis. Seizure 36:70-73

32. Lu VM, Ho Y-T, Nambiar M et al (2018) The perioperative efficacy and safety of antifibrinolytics in adult spinal fusion surgery: a systematic review and meta-analysis. Spine 43:E949-E958

33. Mannucci PM (1998) Hemostatic drugs. N Engl J Med 339:245-253

34. Meybohm P, Kohlhof H, Wirtz DC et al (2019) Präoperative Anämie in der Hüft- und Kniegelenkendoprothetik.ZOrthop Unfall 158:194-200

35. Moore EE, Moore HB, Gonzalez E et al (2016) Rationale for the selective administration of tranexamic acid to inhibit fibrinolysis in the severely injured patien. Transfusion 56(Suppl 2):S110-S114

36. Moskal JT, Capps SG (2016) Meta-analysis of intravenous tranexamic acid in primary total hip arthroplasty. Orthopedics 39:e883-e892

37. Myers SP, Kutcher ME, Rosengart MR et al (2019) Tranexamic acid administration is associated with an increased risk of posttraumatic venous thromboembolism. J Trauma Acute Care Surg $86: 20-27$

38. Napolitano LM (2017) Prehospital tranexamic acid: what is the current evidence? Trauma Surg Acute Care Open 2:e56

39. National Institute for Health Clinical Excellence (2016) Major trauma: assessment and initial management

40. Petersen PB, Kehlet $H$, Jørgensen CC (2018) Safety of in-hospital only thromboprophylaxis after fasttrack total hip and knee arthroplasty: a prospective follow-up study in 17,582 procedures. Thromb Haemost 118:2152-2161

41. Pfizer Pharma PFE GmbH (2018) Fachinformation "Cyklokapron-Injektionslösung". Datum der Erteilung der Zulassung: 19. Dezember 2005, Stand der Information: Mai 2018, https://www.pfizer. de/sites/default/files/FI-8797.pdf. Zugegriffen: 11. Feb. 2021

42. Raza I, Davenport R, Rourke C et al (2013) The incidence and magnitude of fibrinolytic activation in trauma patients. JThromb Haemost 11:307-314

43. Reed MJ, Cooke C, Mcmahon N et al (2020) Improvements in National Code Red transfusion practice in Scotland after adoption of recommendations from the Scottish National Code Red 2015 review. Injury 51:913-918

44. Ripollés-Melchor J, Abad-Motos A, Díez-Remesal Y et al (2020) Association between use of enhanced recovery after surgery protocol and postoperative complications in total hip and knee arthroplasty in the postoperative outcomes within enhanced recovery after surgery protocol in elective total hip and knee Arthroplasty study (POWER2). JAMA Surg 155(4):e196024

45. Roberts I, Shakur H, Afolabi A et al (2011) The importance of early treatment with tranexamic acid in bleeding trauma patients: an exploratory analysis of the CRASH-2 randomised controlled trial.Lancet 377:1096-1101, 1101.e1091-1092

46. Ross D, Erkocak O, Rasouli MRet al (2019) Operative time directly correlates with blood loss and need for blood transfusion in total joint arthroplasty. Arch Bone Joint Surg 7:229-234

47. Roth P, Kaufner L, Treskatsch S et al (2017) Die perioperative Gabe von Tranexamsäure: 
Empfehlung der AE-Deutschen Gesellschaft für Endoprothetik

48. Samama CM (2019) Fast-track procedures in major orthopaedic surgery: is venous thromboembolism prophylaxis still mandatory? Thromb Haemost 119:3-5

49. Samama CM, Afshari A (2018) European guidelines on perioperative venous thromboembolism prophylaxis. Eur J Anaesthesiol 35:73-76

50. Song K, Pan P, Yao Y et al (2019) The incidence and risk factors for allogenic blood transfusion in total knee and hip arthroplasty. J Orthop Surg Res $14: 273$

51. Spahn DR, Bouillon B, Cerny V et al (2019) The European guideline on management of major bleeding and coagulopathy following trauma:fifth edition. Crit Care 23:98

52. Stein P, Studt J-D, Albrecht R et al (2018) The impact of prehospital tranexamic acid on blood coagulation in trauma patients. Anesth Analg 126:522-529

53. Sukeik M, Alshryda S, Haddad FS et al (2011) Systematic review and meta-analysis of the use of tranexamic acid in total hip replacement. J Bone Joint Surg Br 93:39-46

54. Wang C, Xu G-J, Han Z et al (2015) Topical application of tranexamic acid in primary total hip arthroplasty: a systemic review and meta-analysis. Int J Surg 15:134-139

55. Wang F, Zhao K-C, Zhao M-M et al (2018) The efficacy of oral versus intravenous tranexamic acid in reducing blood loss after primary total knee and hip arthroplasty: a meta-analysis. Medicine 97:e12270

56. Watts G (2016) Utako Okamoto. Lancet 387:2286

57. Wirtz MR, Schalkers DV, Goslings JC et al (2020) The impact of blood product ratio and procoagulant therapy on the development of thromboembolic events in severely injured hemorrhaging trauma patients. Transfusion 60:1873-1882

58. Woman Trial Collaborators (2017) Effect of early tranexamic acid administration on mortality, hysterectomy, and other morbidities in women with post-partum haemorrhage (WOMAN): an international, randomised, double-blind, placebocontrolled trial. Lancet 389:2105-2116

59. Yang B, Li H, Wang D et al (2013) Systematic review and meta-analysis of perioperative intravenous tranexamic acid use in spinal surgery. Plos One 8:e55436

60. Yates J, Perelman I, Khair S et al (2019) Exclusion criteria and adverse events in perioperative trials of tranexamic acid: a systematic review and metaanalysis. Transfusion 59:806-824

61. Zhang W, Li N, Chen S et al (2014) The effects of a tourniquet used in total knee arthroplasty: a meta-analysis. J Orthop Surg Res 9:13

62. Zhu J, Zhu Y, Lei P et al (2017) Efficacy and safety of tranexamicacidintotal hip replacement:aPRISMAcompliant meta-analysis of 25 randomized controlled trials. Medicine 96:e9552

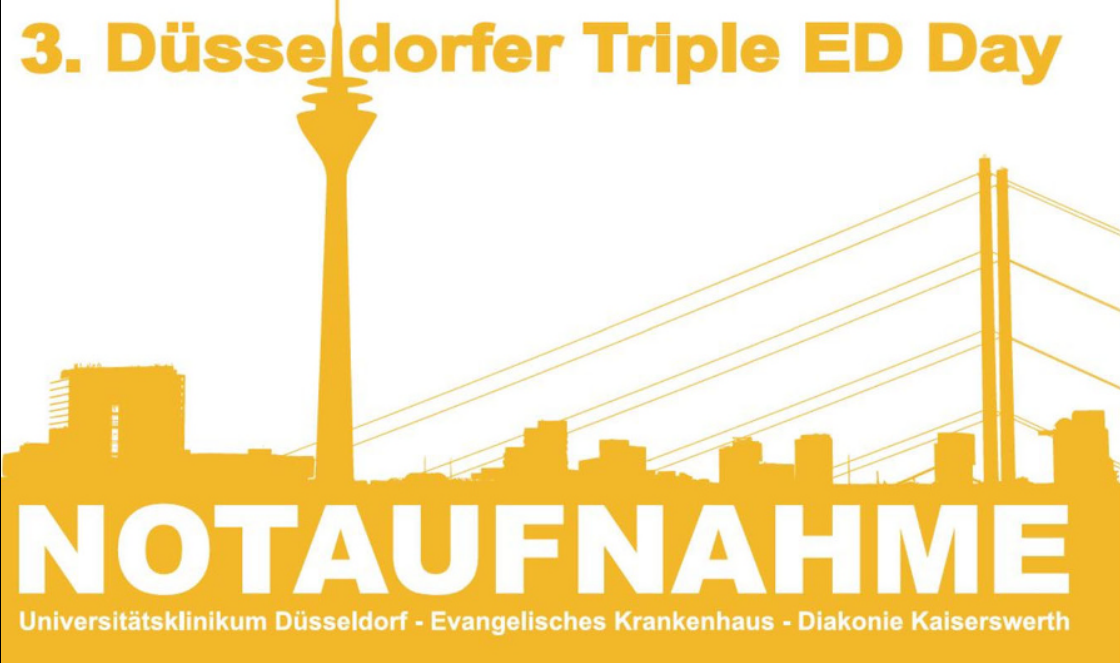

\section{Düsseldorfer „Triple ED Day“ am 11.09.2021}

Regelmäßige Fortbildungen sind wichtig, gerade wenn es um Notfallmedizin geht. Der Düsseldorfer "Triple ED Day" gibt uns die Möglichkeit, eine klinikübergreifende Fortbildung rund um Notfallmedizin und Zentrale Notaufnahmen virtuell zu begehen. Ziel ist es, aktuelles notfallmedizinisches Wissen mit besonderem Fokus auf die Zentrale Notaufnahme praxisorientiert zu vermitteln.

Neben einem Update zur Versorgung einzelner Krankheitsbilder wollen wir dabei insbesondere der aktuellen Entwicklung interdisziplinärer Zentraler Notaufnahmen Rechnung tragen. Da in der Notfallmedizin PatientInnen nicht mit einer Diagnose, sondern mit einem Symptom vorstellig werden, verfolgen wir in unserer Fortbildung einen leitsymptom- und fallorientierten Ansatz.

Da Notfallmedizin nur im Team funktionieren kann, laden wir neben den in den Zentralen Notaufnahmen tätigen pflegerischen und ärztlichen Mitarbeitenden auch Kolleginnen und Kollegen aller klinischen Partner und die Mitarbeitenden des Rettungs- und Notarztdienstes ein. Notfallmedizinisch interessierte Studierende sind ebenfalls herzlich willkommen. Durch die Chatfunktion des OnlineFormates ergibt sich Gelegenheit für Fragen und spannende Diskussionen der Vorträge. Wir würden uns sehr freuen, Sie bei unserer interprofessionellen und interdisziplinären Veranstaltung online begrüßen zu dürfen!
Wissenschaftliche Leitung:

Prof. Dr. Michael Bernhard, Düsseldorf

Alexander Kleophas, Düsseldorf

Martin Pin, Düsseldorf

\section{Organisation:}

Universitätsklinikum Düsseldorf

Zentrale Notaufnahme

Moorenstr. 5

40225 Düsseldorf

\section{Teilnahme:}

Der Düsseldorfer Triple ED Day wird über die bekannte Fortbildungsplattform NOW TO GO übertragen.

Teilnehmen kann jeder, der sich kostenlos unter www.nowtogo.de registriert hat.

Die Veranstaltung ist kostenfrei. Die Anerkennung der Fortbildungsveranstaltung wurde bei der Ärztekammer Berlin und bei der Registrierung berufl ich Pflegender beantragt. 